ASEAN Journal of Chemical Engineering 2021, Vol. 21, No. 2, 133-142

\title{
Enhancement of Delignification and Glucan Bontent of Sugarcane Bagasse by Alkali Pretreatment for Bioethanol Production
}

Kyaw Wunna *1,3

Kiohiko Nakasaki ${ }^{2}$

Joseph Auresenia ${ }^{1}$

Leonila Abella ${ }^{1}$

Pag-asa Gaspillo ${ }^{1}$

${ }^{1}$ Chemical Engineering Department, De La Salle University-Manila, Manila, Philippines

${ }^{2}$ Department of International Development Engineering, Tokyo Institute of Technology, Tokyo, Japan

${ }^{3}$ Department of Industrial Chemistry, Yadanabon University, Mandalay, Myanmar

*e-mail: kyawwunna550@gmail.com

Submitted 26 August 2020

Revised 10 September 2021 Accepted 14 September 2021

Abstract. The current work aimed to enhance the delignification of sugarcane bagasse (SCB) for bioethanol production. The optimization of alkali (sodium hydroxide) pretreatment parameters such as concentration and residence time was carried out by the Taguchi method using $\mathrm{L}_{16}$ orthogonal array with two factors and four levels. Sugarcane bagasse powder was mixed with sodium hydroxide $(\mathrm{NaOH})$ solution $(0.5-2 \mathrm{wt} . \%)$ and heated in an autoclave at $121^{\circ} \mathrm{C}$ and at varied times (30-120 min). From the statistical analysis of data, it was observed that delignification and glucan increased with the increased concentration and short time. The optimum parameters of $\mathrm{NaOH}$ pretreatment were $2 \mathrm{wt}$.\% of $\mathrm{NaOH}$ concentration and 30 minutes of residence time. At the optimum conditions, $86.8 \%$ delignification and $46.6 \%$ glucan content of SCB were obtained. Thus, alkali pretreatment optimized by Taguchi design is the effective method to remove lignin and to increase cellulose or glucan content in sugarcane bagasse for facilitating the further catalytic hydrolysis in bioethanol production.

Keywords: Delignification, Glucan, Lignin, Sugarcane bagasse, Alkali pretreatment

\section{INTRODUCTION}

Sugarcane bagasse $(\mathrm{SCB})$ is one of the largest cheap lignocellulosic materials among various agricultural residues and it is a waste of the sugar juice extraction process (Ju et al. 2011). Current uses of SCB are energy sources in sugar mills and distillery plants, generation of electricity to be sold to the national grid (Rocha et al. 2011), pulp and paper production, phenolic compounds, polymer films, fertilizer and pesticides, ethanol, butanol, furfural, natural adhesive resin, xylitol, nanocomposites, animal feeds and other products (Ju et al. 2011). However, a major part of the bagasse in the sugar mill remained underutilized (Rocha et al. 2011).

Sugarcane bagasse is a kind of herbaceous lignocelluloses (Wang et al. 2020). SCB is mainly composed of cellulose, 
134 Enhancement of Delignification and Glucan Content of Sugarcane Bagasse by Alkali Pretreatment for Bioethanol Production

hemicellulose, and lignin. Among the compositions, carbohydrate, lignin, extractives, and ash were $61-63 \%, 25-28 \%, 5-$ $6 \%$, and $6-7 \%$ respectively (Jonglertjunya et al. 2014). Cellulose and hemicellulose as the major carbohydrates play vital roles in bioethanol production through hydrolysis converting into glucose and xylose (Jung et al. 2018). The carbohydrates of dried solid contain $40-50 \%$ cellulose composed of several glucose units and include a crystalline structure. The second-largest portion of SCB is hemicelluloses (25-35\%) which are composed of an amorphous polymer. In hemicelluloses, xylose exists as major pentose sugar, and small units of galactose, arabinose, glucose, and mannose are also found. The remained portion is mostly lignin (10-14\%) and other traces of compounds.

Of all constituents in $\mathrm{SCB}$, lignin is the most difficult one to decompose and is more impervious to any biological degradation than cellulose and hemicellulose. Lignin is a biopolymer derived from plants and wood (Clavo-Flores et al. 2015). The lignin of herbaceous biomass like SCB mainly consists of the three major phenylpropane units, namely guaiacyl $(G)$, p-hydroxyphenyl $(H)$, and syringcyl (S) (Wang et al. 2020). The chemical arrangement of lignin bonds obtained the lignin degradation slowly at a temperature from low to high $\left(100-900^{\circ} \mathrm{C}\right)$ (Jonglertjunya et al. 2014). Moreover, lignin is considered a distinctive naturally available renewable resource for the manufacture of aromatic chemicals (Long et al. 2013). Although there are several methods in the determination of lignin, the controversy remains to characterize the lignin type because of different issues such as the origin of lignin, plant species, analytical techniques, and isolation methods. The oldest and most common method is the gravimetric isolation using mineral acid which derives the solid residue lignin, also known as Klason lignin (Clavo-Flores et al. 2015).

Due to its complex structures and nonproductive binding, the pretreatment step to remove the lignin component of the biomass material is a crucial step in the bioconversion process. However, the pretreatment method is an important step and has higher costs in bioconversion of cellulosic biomass for their applications in biorefinery concepts. Several pretreatments methods such as chemical, physical and physico-chemical pretreatment for different kinds of lignocellulosic materials have been reported by several researchers (Singh et al. 2014, Rocha et al. 2011, Asgher et al. 2013, Jung et al. 2018, Wang et al, 2020). Among several pretreatment methods, pretreatment with alkali has been considered as an effective way for pretreating different lignocellulosic materials to increase the removal of lignin and to enhance the glucan content in pretreated residue, which makes the easier saccharification to obtain a high concentration of glucose (Wang et al. 2020, Kim et al. 2016, Jung et al. 2018, Gao et al. 2013). Glucose is also the starting material for many important chemicals like furfural, 5hydroxy methyl furfural, and 2, 5furandicarboxilic acid (Radhakumari et al. 2014) aside from ethanol production. Alkali pretreatment is also claimed to be greater potential for industrial application due to its mild processing conditions (Kim et al. 2016, Jung et al. 2018).

Main reactions of alkali pretreatment occur as decomposition of lignin and hemicellulose, and saponification of intermolecular of ester bonds, enhancing the porosity of lignocellulosic biomass (Kim et al. 2016, Hosgun et al. 2017). Alkali pretreatments are usually performed with 
alkali reagents such as sodium hydroxide $(\mathrm{NaOH})$, potassium hydroxide $(\mathrm{KOH})$, lime, or ammonium hydroxide. $\mathrm{NaOH}$ is considered as the most effective chemical because of its cheaper price, higher solubility, and stronger alkalinity (Kim et al. 2016, Hosgun 2017, Wang et al. 2019). While performing the pretreatment, $\mathrm{NaOH}$ may cause bond cleavage between carbohydrates and lignin, resulting in the dissolution of lignin in $\mathrm{NaOH}$ (Jung et al. 2018). Wang et al. (2020) revealed that lignin removal by $\mathrm{NaOH}$ pretreatment was found to be easier in herbaceous biomass than in hardwood and softwood, and $\mathrm{NaOH}$ pretreatment could remove $70 \%$ lignin in wheat straw, corn straw, and SCB.

However, there remains a challenge in alkali pretreatment of SCB with sodium hydroxide to refrain from the delignification of SCB during pretreatment using an appropriate design of experiment. It is important to optimize the $\mathrm{NaOH}$ pretreatment parameters using the most robust design so that it is a more adaptable and practical technology for industrial application and improvement of economic feasibility. Systematical optimization of $\mathrm{NaOH}$-pretreatment is performed with the design of experiments (DOE) and includes the determination of the interactions between the main parameters in the pretreatment. There are several methods of DOE in which the full factorial method is the most complete one. However, it is expensive, a large number of experiments, and time-consuming. Instead, fractional factorial designs are being used because they minimize the size of experiments, time, and costs of the experimental run. The Taguchi method is one of the simple and robust designs used for DOE (Darvishi and Moghaddami 2019). The main purpose of the Taguchi DOE approach is to reduce the cost and experimental run without altering the quality of products with improved process performance showing its higher robustness (Das et al. 2016).

The present work was to focus on the sodium hydroxide pretreatment of SCB to highly remove the lignin, and enhance the glucan content from SCB for subsequent use in the fermentation process of bioethanol. In this treatment, concentrations of sodium hydroxide $(\mathrm{NaOH})$ and residence time at constant temperature $\left(121^{\circ} \mathrm{C}\right)$ were varied to determine their effect on the removal of lignin and enhancement of glucan from SCB using $\mathrm{L}_{16}$ orthogonal array of Taguchi DOE method that would contribute to more feasible and applicable pretreatment method.

\section{MATERIALS AND METHOD}

\section{Preparation and Characterization of Sample}

SCB was obtained from markets in Yangon, Myanmar. All chemicals including $\mathrm{NaOH}$, glucose and sulfuric acid used in this research were analytical grade and purchased from Wako Chemicals, Japan. The sample was cleaned with running water and oven-dried at $65^{\circ} \mathrm{C}$. The dried sample was then milled in Ultra Centrifugal Miller ZM 10 (Japan) to obtain less than $0.5 \mathrm{~mm}$ in size and characterizations of raw SCB were carried out as described in our previous studies (Wunna et al. 2017). The moisture content of the dried SCB was $3.6 \%$ and the lignin, glucan, and xylan content were $25.4,40.2$, and $22.5 \%$ respectively.

\section{Alkali Pretreatment of SCB}

Sodium hydroxide pretreatment was involved with different concentrations (0.52.0 wt.\%) of $\mathrm{NaOH}$ solution. The solution was added into $1 \mathrm{~g}$ of the dried sample as $10 \mathrm{wt} . \%$ 
136 Enhancement of Delignification and Glucan Content of Sugarcane Bagasse by Alkali Pretreatment for Bioethanol Production

solid-liquid ratios and heated in an autoclave at $121^{\circ} \mathrm{C}$ and at a varied time $(30-120 \mathrm{~min})$ according to Table 1 . After heating, rapid cooling of the sample was done to reach room temperature and vacuum filtration was carried out to obtain solid residue and liquid. The solid residue was then neutralized with distilled water and dried as mentioned before.

\section{Analytical Method}

Analysis of dried pretreated solid was conducted based on NREL methods (Sluiter et al. 2011) as mentioned in our previous study (Wunna et al. 2017) and glucose, xylose, and lignin were measured. Percentages of glucan, lignin, and delignification were calculated by Eq. (1) to Eq. (4) based on previous literature (Sluiter et al. 2011, Gao et al. 2013). Lignin is the component combining acid-insoluble lignin (AIL) and acid-soluble lignin (ASL).

$$
\begin{aligned}
& \mathrm{G}=\frac{A \times V}{W \times 1.1} \times 100 \\
& \% \mathrm{AIL}=\frac{W_{A}-W_{B}}{W} \times 100 \\
& \% \mathrm{ASL}=\frac{U V_{A b s} \times V \times D R}{\varepsilon \times W \times p} \times 100 \\
& \mathrm{DL}=\frac{L-[W \times(A I L+A S L)]}{W} \times 100
\end{aligned}
$$

\section{Optimization Using Taguchi Method}

The Taguchi method is based on orthogonal arrays, which is a robust design of the experiment method. It gives a set of the minimum number of experimental runs along with finite data about the significance of factors involved in the process parameter. In this study, the Taguchi method was carried out according to Table 1 where $X_{1}$ is alkali concentration (wt.\%), $X_{2}$ is residence time, $Y_{1}$ is delignification (wt.\%) and $Y_{2}$ is glucan (wt.\%). In the optimization of the pretreatment parameter, the signal-to-noise ratio $(\mathrm{S} / \mathrm{N})$, termed as the logarithmic transformation of results of desired performance, is used as the desired function of the optimization process. There are three criteria to be considered in assigning the $\mathrm{S} / \mathrm{N}$ ratio to determine the optimum response parameter: (1) larger is the best, (2) nominal is the best and (3) smaller is the best (Radhakumari et al. 2014).

Table 1. Design Matrix for Taguchi method to optimize the parameters of delignification process of SCB

\begin{tabular}{ccccc}
\hline Run & \multicolumn{2}{c}{ Variables } & \multicolumn{2}{c}{ Response } \\
\cline { 2 - 5 } No. & $\mathrm{X}_{1}$ & $\mathrm{X}_{2}$ & $\mathrm{Y}_{1}$ & $\mathrm{Y}_{2}$ \\
\hline 1 & 0.5 & 30 & 71.7 & 29.0 \\
2 & 0.5 & 60 & 67.4 & 33.3 \\
3 & 0.5 & 90 & 43.2 & 32.0 \\
4 & 0.5 & 120 & 42.7 & 27.9 \\
5 & 1.0 & 30 & 77.3 & 37.6 \\
6 & 1.0 & 60 & 82.7 & 44.9 \\
7 & 1.0 & 90 & 71.3 & 37.0 \\
8 & 1.0 & 120 & 69.5 & 33.4 \\
9 & 1.5 & 30 & 87.3 & 37.7 \\
10 & 1.5 & 60 & 87.8 & 37.8 \\
11 & 1.5 & 90 & 81.1 & 40.1 \\
12 & 1.5 & 120 & 68.4 & 29.6 \\
13 & 2.0 & 30 & 86.8 & 46.6 \\
14 & 2.0 & 60 & 83.7 & 37.7 \\
15 & 2.0 & 90 & 84.6 & 40.7 \\
16 & 2.0 & 120 & 73.9 & 35.7 \\
\hline
\end{tabular}

Our main goals are to maximize the delignification and glucan content in SCB. Therefore, the 'larger is the best' criterion was chosen to optimize the pretreatment parameters and $\mathrm{S} / \mathrm{N}$ ratio was calculated as Eq. (5): 


$$
\frac{S}{N}=10 \log _{10}\left[\frac{1}{n_{j}} \sum_{j=1}^{n} \frac{1}{Y_{i}^{2}}\right]
$$

where $\mathrm{i}$ is the experiment number, $\mathrm{n}$ is the number of replication ' $i$ ', $j$ is the number of replicates and $Y$ is the performance parameter. Analysis of variance (ANOVA) and $\mathrm{S} / \mathrm{N}$ ratio analysis was done in Minitab $17^{\circ}$ software.

\section{RESULTS AND DISCUSSION}

Pretreatment plays a crucial role in bioethanol production from lignocellulosic materials. In this work, optimization of alkali pretreatment was approached not only to reduce the lignin but to improve glucan in SCB according to $L_{16}$ orthogonal array of Taguchi method since it could reduce the number of experiments, costs of experimental run and time consuming compared to the other full factorial designs (Darvishi and Moghaddami 2019). The design matrix and observed data were listed in Table 1.

\section{Effect of parameters - $\mathrm{S} / \mathrm{N}$ ratios}

In optimization of pretreatment parameters, the signal to noise ratio $(\mathrm{S} / \mathrm{N})$ i.e., the logarithmic transformation of mean squared deviation (MSD) of the desired response, is the better way to accommodate the analysis of result data. Among three criteria, the "larger is best" criterion was chosen to calculate $\mathrm{S} / \mathrm{N}$ ratio for both responses $Y_{1}$ and $Y_{2}$ as the aim is to increase delignification and to enhance glucan of SCB. $\mathrm{S} / \mathrm{N}$ ratios at each level for all the factors are shown in Table 2.

The highest value of $\mathrm{S} / \mathrm{N}$ ratio indicates both maximum delignification and glucan in SCB and their main effect plots are shown in figures 1 and 2. Ranks were assigned based on the delta values of each factor subtracting the lowest value of $\mathrm{S} / \mathrm{N}$ ratio from the highest one. The higher the difference in $\mathrm{S} / \mathrm{N}$ ratio of each factor, the higher is its influence on delignification and glucan.

Table 2. Response table for $\mathrm{S} / \mathrm{N}$ ratios corresponding to $Y_{1}$ and $Y_{2}$

\begin{tabular}{ccccc}
\hline \multirow{2}{*}{ Level } & \multicolumn{2}{c}{$\mathbf{Y}_{\mathbf{1}}$} & \multicolumn{2}{c}{$\mathbf{Y}_{\mathbf{2}}$} \\
\cline { 2 - 5 } & $\mathrm{X}_{1}$ & $\mathrm{X}_{2}$ & $\mathrm{X}_{1}$ & $\mathrm{X}_{2}$ \\
\hline 1 & 34.75 & 38.12 & 29.68 & 31.41 \\
2 & 37.50 & 38.06 & 31.60 & 31.64 \\
3 & 38.14 & 36.62 & 31.14 & 31.43 \\
4 & 38.29 & 35.88 & 32.03 & 29.97 \\
Delta & 3.54 & 2.24 & 2.36 & 29.97 \\
Rank & 1 & 2 & 1 & 2 \\
\hline
\end{tabular}

From Table 2, it is clear that the factor concentration was presenting a positive effect on $Y_{1}$ indicating that the higher the concentration of alkali, the better was its performance. In contrast, reaction time was showing a lower effect on $Y_{1}$ leading to that decrease in time resulting in increased performance. Therefore higher alkali concentration enhanced the delignification of SCB with a short duration. It is possible that strong alkali $(\mathrm{NaOH})$ disrupts the cross-ester linkages between lignin and structural carbohydrates especially hemicellulose, resulting in the degradation of lignin and hemicellulose and saponification of ester bonds (Kim et al. 2016, Hosgun et al. 2017, Jung et al. 2018). This further contributes to higher glucan content in pretreated solid residue. Jung et al. (2018) reported that higher delignification and glucan content of switchgrass was achieved with higher $\mathrm{NaOH}$ concentration and longer time using full factorial design. Therefore, this study could save processing time, leading to less energy consumption. In addition, $\mathrm{NaOH}$ pretreated SCB gave higher glucan content and lignin removal than other pretreatment methods 
138 Enhancement of Delignification and Glucan Content of Sugarcane Bagasse by Alkali Pretreatment for Bioethanol Production

like liquid hot water (LHW), which has high power consumption, and $\mathrm{HCl}$ (Gao et al. 2013).

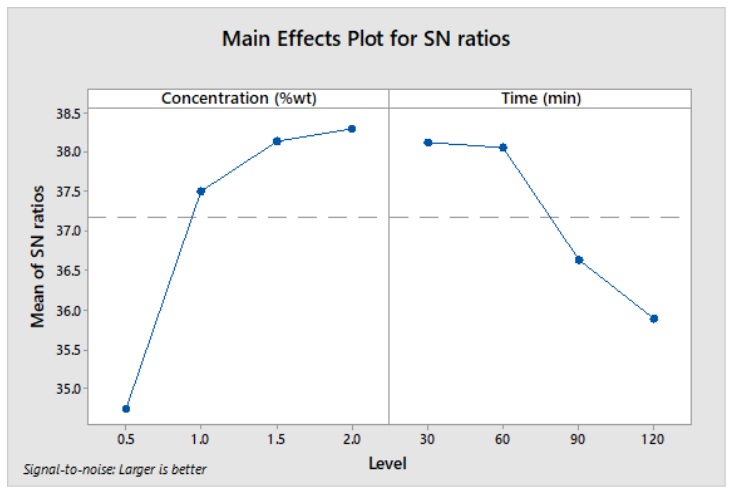

(a)

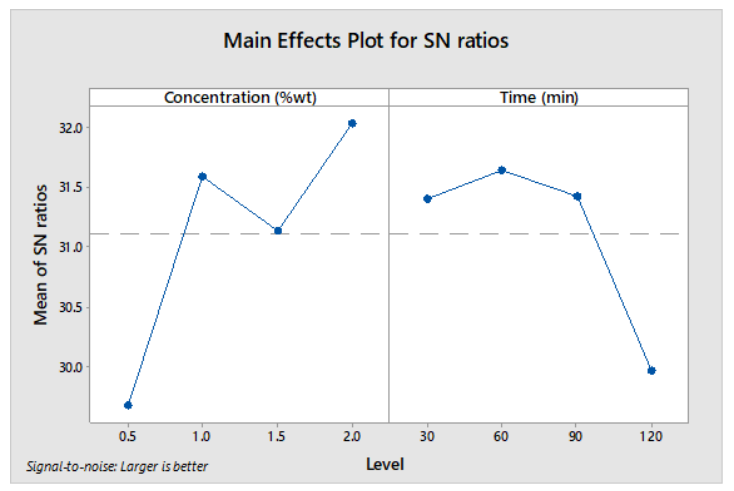

(b)

Fig. 1: Main effect plots for $S / N$ ratio of (a) delignification $\left(\mathrm{Y}_{1}\right)$ and $(\mathrm{b})$ glucan $\left(\mathrm{Y}_{2}\right)$

An unusual trend was observed in the performance of $\mathrm{Y}_{2}$ with respect to the concentration factor. As can be seen in Figure $1(b), S / N$ ratio of concentration increased from level 1 to 2 and decreased to level 3, and then increased to level 4 . This could be due to the fact that a long time with a certain temperature further degraded the glucan into undesirable products (Palmqvist and Hahn-Hagerdal 2000). However, the concentration of alkali has a larger effect on the yield of glucan followed by time according to rank values. The highest $\mathrm{S} / \mathrm{N}$ ratio corresponding to delignification and glucan of SCB illustrated that the best combination of parameters was level 4 of alkali concentration and level 1 of time (i.e
$2.0 \%$ wt. and $30 \mathrm{~min}$ ) when the temperature was fixed at $121^{\circ} \mathrm{C}$ for all experiments.

\section{Effect of parameters - the analysis of variance (ANOVA)}

The analysis of variance (ANOVA) was performed using observed data and is presented in Tables 3 (a) and (b). It revealed that all the factors were a statistically significant effect on both $Y_{1}$ and $Y_{2}$ except from time in the case of $Y_{2}$ as $95 \%$ confidence interval and $(p<0.05)$ were chosen. It was also observed that the F-value of alkali concentration was higher than that of time, confirming its highest influence on the delignification process as well as enhancement of glucan in SCB.

Table 3(a). Analysis of variance corresponding to $\mathrm{Y} 1$

\begin{tabular}{cccccc} 
& \multicolumn{5}{c}{ Y1 } \\
\cline { 2 - 6 } Source & DF & Adj SS & $\begin{array}{c}\text { Adj } \\
\text { MS }\end{array}$ & $\begin{array}{c}\text { F- } \\
\text { Value }\end{array}$ & $\begin{array}{c}\text { P- } \\
\text { Value }\end{array}$ \\
\hline $\mathrm{X}_{1}$ & 3 & 1741.4 & 580.5 & 15.9 & 0.001 \\
$\mathrm{X}_{2}$ & 3 & 839.1 & 279.7 & 7.7 & 0.008 \\
Residual & 9 & 328.9 & 36.5 & & \\
Error & & & & & \\
Total & 15 & & & \\
\hline
\end{tabular}

Table 3(b). Analysis of variance corresponding to $Y_{2}$

\begin{tabular}{cccccc}
\hline & \multicolumn{5}{c}{$\mathbf{Y}_{\mathbf{2}}$} \\
\cline { 2 - 6 } Source & DF & $\begin{array}{c}\text { Adj } \\
\text { SS }\end{array}$ & $\begin{array}{c}\text { Adj } \\
\text { MS }\end{array}$ & $\begin{array}{c}\text { F- } \\
\text { Value }\end{array}$ & $\begin{array}{c}\text { P- } \\
\text { Value }\end{array}$ \\
\hline$X_{1}$ & 3 & 207.1 & 69.0 & 6.1 & 0.015 \\
$X_{2}$ & 3 & 118.0 & 39.3 & 3.5 & 0.064 \\
Residual & 9 & 102.1 & 11.3 & & \\
$\quad$ Error & & & & & \\
Total & 15 & & & & \\
\hline
\end{tabular}

Regression analysis was done based on the observed data on delignification as well as glucan and summarized in Tables 4 (a) and (b). 
Table 4(a). Regression analysis

corresponding to $\mathrm{Y} 1$

\begin{tabular}{cccccc}
\hline & \multicolumn{5}{c}{ Y1 } \\
\cline { 2 - 6 } Source & DF & $\begin{array}{c}\text { Adj } \\
\text { SS }\end{array}$ & $\begin{array}{c}\text { Adj } \\
\text { MS }\end{array}$ & $\begin{array}{c}\text { F- } \\
\text { Value }\end{array}$ & $\begin{array}{c}\text { P- } \\
\text { Value }\end{array}$ \\
\hline $\mathrm{R}$ & 2 & 2173 & 1087 & 19.2 & 0.000 \\
$\mathrm{X}_{1}$ & 1 & 1410 & 1410 & 24.9 & 0.000 \\
$\mathrm{X}_{2}$ & 1 & 763.8 & 763.8 & 13.5 & 0.003 \\
Error & 13 & 736 & 56.6 & & \\
Total & 15 & 2909 & & & \\
\hline
\end{tabular}

Table 4(b). Regression analysis corresponding to $\mathrm{Y} 1$

\begin{tabular}{cccccc}
\hline & \multicolumn{5}{c}{ Y1 } \\
\cline { 2 - 6 } Source & DF & $\begin{array}{c}\text { Adj } \\
\text { SS }\end{array}$ & $\begin{array}{c}\text { Adj } \\
\text { MS }\end{array}$ & $\begin{array}{c}\text { F- } \\
\text { Value }\end{array}$ & $\begin{array}{c}\text { P- } \\
\text { Value }\end{array}$ \\
\hline $\mathrm{R}$ & 2 & 219 & 109.5 & 6.8 & 0.009 \\
$\mathrm{X}_{1}$ & 1 & 145.3 & 145.3 & 9.1 & 0.010 \\
$\mathrm{X}_{2}$ & 1 & 73.7 & 73.7 & 4.6 & 0.051 \\
Error & 13 & 208.2 & 16.02 & & \\
Total & 15 & 427.2 & & & \\
\hline
\end{tabular}

The equations resulted are as follows:

$Y_{1}=68.17+16.79 X_{1}-0.2060 X_{2}$

$\mathrm{R}^{2}=74.70 \%$

$Y_{2}=34.38+5.39 X_{1}-0.64 X_{2}$

$R^{2}=51.26 \%$

The model equations fitted with experimental data are plotted as shown in figures 2(a) and 2(b). From the contour plots, it can be concluded that maximum delignification appeared at 1-2\% alkali concentration and at $30-90 \mathrm{~min}$, and the maximum glucan percentage at $1 \%$ alkali concentration and 50-60 min.

From the statistical analysis of the observed data, it was concluded that the delignification of SCB increased with the increase of alkali concentration and decreased time. It indicates that delignification and glucan do not vary with prolonged time. This result demonstrates that alkali concentration plays important role in the removal of more lignin from SCB than processing time. It can be explained that alkaline conditions make the solubilization of some phenolic compounds attached to cell wall components (i.e polysaccharides and lignin), resulting from the decomposition of $\alpha$-ester bonds in lignin-polysaccharide complexes due to the nature of ester linkages (Ju et al. 2011).

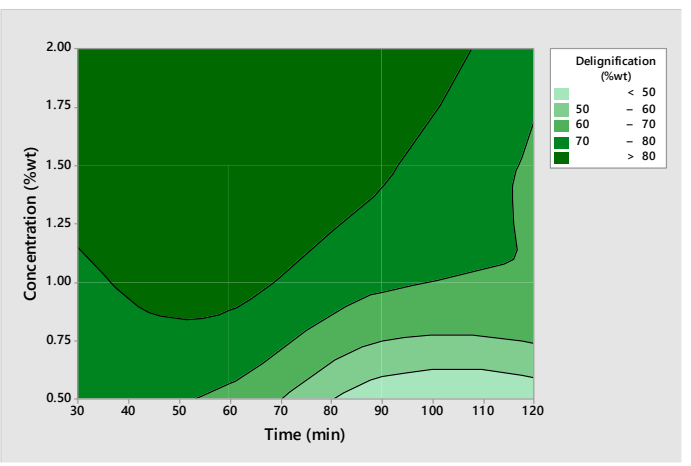

(a)

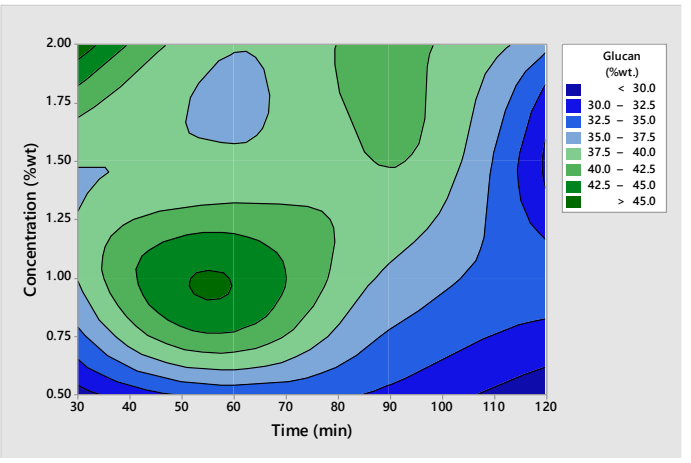

(b)

Fig. 2: Contour plots for (a) delignification $\left(\mathrm{Y}_{1}\right)$ and $(\mathrm{b})$ glucan $\left(\mathrm{Y}_{2}\right)$

Furthermore, cellulose has less potential for solubilization on pretreatment. On the other hand, cellulose cannot be easily hydrolyzed by alkali pretreatment because it is known to be a much more recalcitrant and surface-governed reaction mechanism (Canilha et al. 2011). Xu et al. (2012) and Ahgher et al. (2013) stated that mild alkali treatment conditions completely converted cellulose I into cellulose II, indicating that it is a more stable form with antiparallel chain structure in $\mathrm{NaOH}$ solution. However, 
140 Enhancement of Delignification and Glucan Content of Sugarcane Bagasse by Alkali Pretreatment for Bioethanol Production

processing time should be enough to break down bonds present in lignin-polysaccharide complexes. Moreover, the achievement of alkali pretreatment lies in the lignin content in the substrate (Ju et al. 2011).

According to overall data analysis, the optimum conditions of $\mathrm{NaOH}$ pretreatment were 2 wt.\% of $\mathrm{NaOH}$ concentration (level 4) and at $30 \mathrm{~min}$ (level 1). At optimum conditions, $86.8 \%$ delignification and $46.6 \%$ glucan content of SCB were obtained. Asgher et al. (2013) reported maximum delignification (48.7\%) of SCB was obtained by $5 \% \mathrm{NaOH}$ treatment for $30 \mathrm{~min}$ at $121^{\circ} \mathrm{C}$. Liu et al. (2016) investigated the pretreatment of corn stover using high concentration (12 wt.\%) at $140^{\circ} \mathrm{C}$ for $20 \mathrm{~min}$, and lignin could be reduced by $18.5 \%$ to $7.4 \%$ and glucose increased 36.7 to $65.0 \%$, respectively. Low consumption of $\mathrm{NaOH}$ in alkali pretreatment could probably minimize the use of wash water and generation of wastewater after pretreatment which are the current bottleneck problems and are considered as another factor in further research. On the other hand, black liquor should be recycled in another pretreatment. In another recent study (Wang et al. 2020), 72.1\% delignification and $56.3 \%$ glucan content of the pretreated SCB were achieved by $2 \%$ $(\mathrm{m} / \mathrm{v})$ for $120 \mathrm{~min}$ at $80^{\circ} \mathrm{C}$ with a $10 \%(\mathrm{~m} / \mathrm{v})$ solid-liquid ratio. Compared to previous literature, the $\mathrm{NaOH}$ pretreatment of SCB using the Taguchi method shows the best performance in terms of delignification and the comparable performance in terms of glucan content.

\section{CONCLUSIONS}

The alkali $(\mathrm{NaOH})$ pretreatment could enhance the delignification and glucan content of SCB by using the Taguchi method.
The optimum parameters of alkali pretreatment of SCB were $\mathrm{NaOH}$ concentration of $2 \mathrm{wt} . \%$ and residence time of 30 min. It was found that $\mathrm{NaOH}$ concentration was the most influenced parameter in the alkali pretreatment of SCB. Enhanced delignification and glucan content can help the further catalytic hydrolysis and make the easier saccharification to obtain high sugar concentration as well as high ethanol concentration through the fermentation process. Additionally, the use of the Taguchi method in the optimization of alkali pretreatment provides an effective procedure to find the optimum condition. However, to more understand the alkali delignification process in bioethanol production, the changes of lignin chemistry in solid residue and liquid fraction should be further studied.

\section{ACKNOWLEDGEMENT}

The authors would like to express their sincere thanks to AUN/SEED-Net and JICA for their financial support of this work.

\section{NOMENCLATURE}

$\begin{array}{lll}G & : & \text { Glucan [wt.\%] } \\ D L & : & \text { Deligninfication [wt.\%] } \\ L & : & \text { Initial lignin [wt.\%] } \\ W_{A} & : & \text { Weight of acid-insoluble } \\ & & \text { sample [g] } \\ W_{B} & : & \text { Weight of acid-insoluble ash [g] } \\ W & : \text { Weight of dried sample [g] } \\ A I L & : \text { Acid insoluble lignin [wt.\%] } \\ A S L & : \text { Acid soluble lignin [wt.\%] } \\ V & : \text { Volume of sample solution [L] } \\ D R & & \text { Dilution rate } \\ \varepsilon & : \text { Absorptivity of biomass at a } \\ & \quad \text { specific wavelength } \\ p & : \text { Path length [mm] }\end{array}$




\begin{tabular}{lll}
\hline$U V_{A b s}:$ & UV absorbance \\
$S / N$ & $:$ & Signal to Noise ratio \\
$X_{1}$ & $:$ & Concentration of $\mathrm{NaOH}$ [wt.\%] \\
$X_{2}$ & $:$ & Time [min] \\
$Y_{1}, Y_{2}:$ & $:$ Response [Delignification and \\
& & glucan, respectively] \\
$D F$ & $:$ & Degree of freedom \\
$A d j S S:$ & $:$ Adjusted Sum of Square \\
$A d j M S:$ & Adjusted Mean Square \\
$F$ & $:$ Variance Ratio \\
$P$ & $:$ Level of Significance
\end{tabular}

\section{REFERENCES}

1. Asgher, M., Ahmad, Z., Iqbal, H.M.N. (2013). "Alkali and enzymatic delignification of sugarcane bagasse to expose cellulose polymers for saccharification and bioethanol production," Inds. Crops \& Products, 44, 488-495.

2. Calvo-Flores F. G., Dobado, J. A., IsacGarcia, J., Martin-Martinez, F. J. (2015). Lignin and lignans as renewable raw materials, John Wiley \& Sons, New York, U.S.A.

3. Canilha, L., Santos, V.T.O., Rocha, G.J.M., Almeida e Silva, J.B., Silva, S.S., Felipe, M.G.A., Ferraz, A., Milagres A.M.F., Carvalho W. (2011). "A study on the pretreatment of a sugarcane bagasse sample with dilute sulfuric acid," J. Ind. Microbiol. Biotechnol, 38, 1467-1475.

4. Darvishi, F., and Moghaddami N. A. (2019). "Optimization of an industrial medium form molasses for bioethanol production using the Taguchi statistical experimental-design method," Fermentation, 5, 14.

5. Das, S. P., Gupta, A., Das, D., Goyal, A. (2016). "Enhanced bioethanol production from water hyacinth (Eichhornia crassipes) by statistical optimization of fermentation process parameters using Taguchi orthogonal array design," Int. Biodet. \& Biodegr., 109, 174-184.

6. Gao Y., Xu J., Zhang Y., Yu Q., Yuan Z., Liu Y. (2013). "Effects of different pretreatment methods on chemical composition of sugarcane bagasse and enzymatic hydrolysis," Bioresour. Technol., 144, 396-400.

7. Hosgun, E. Z., Berikten, D., Kivanc, M., Bozan B. (2017). "Ethanol production from hazelnut shell through enzymatic saccharification and fermentation by low-temperature alkali pretreatment," Fuel, 196, 280-287.

8. Jonglertjunya W., Juntong, T., Pakkang, N., Srimarut, N., Sakdaronnarong C. (2014). "Properties of lignin extracted from sugarcane bagasse and its efficacy in maintaining postharvest quality of limes during storage," LWT - Food Sci. \& Technol., 57, 116-125.

9. Ju, Y. H., Huynh, L. H., Kasim N. S., Guo, T. J., Wang J. H., Fazary, A. E. (2011). "Analysis of soluble and insoluble fractions of alkali and subcritical water treated sugarcane bagasse," Carbohydr. Polym, 83, 591-599.

10. Jung, W., Savithri, D., Sharma-Shivappa, R., Kolar P. (2018). "Changes in lignin chemistry of switchgrass due to delignification by sodium hydroxide pretreatment," Energies, 11, 276.

11. Kim, J. S., Lee, Y. Y., Kim, T. H. (2016). "A review on alkaline pretreatment technology for bioconversion of lignocellulosic biomass," Bioresour. Technol., 199, 42-48.

12. Liu, H., Pang, B., Zhou, J., Han, U., Lu, J., $\mathrm{Li}, \mathrm{H}$. (2016). "Comparative study of pretreated corn stover for sugar production using cotton pulping black 
142 Enhancement of Delignification and Glucan Content of Sugarcane Bagasse by Alkali Pretreatment for Bioethanol Production

liquor (CBPL) instead of sodium hydroxide," Ind. Crops Prod., 84, 97-103.

13. Long, J., Li, X., Guo, B., Wang, L., Zhang N. (2013). "Catalytic delignification of sugarcane bagasse in the presence of acidic ionic liquids," Catalysis Today, 200, 99-105.

14. Palmqvist, E., Hahn-Hagerdal, B. (2000). "Fermentation of lignocellulosic hydrolysates. II: inhibitors and mechanisms of inhibition," Bioresour. Technol., 74, 25-33.

15. Radhakumari, M., Ball, A., Bhargava, S. K., Satyavathi, B. (2014). "Optimization of glucose formation in karanja biomass hydrolysis using Taguchi robust method," Bioresour. Technol., 166, 534540.

16. Rocha, G. Jackson de Moraes, Martin C., Soares, I. B., Maior, A. M. S., Baudel, H. M., Moraes de Abreu, C. A. (2011). "Dilute mixed-acid pretreatment of sugarcane bagasse for ethanol production," Biomass \& Bioenergy, 35, 663-670.

17. Singh, R., Shukla, A., Tiwari, S., Srivastava, M. (2014). "A review on delignification of lignocellulosic biomass for enhancement of ethanol production potential," Renewab. Sust. Ener. Reviews, 32, 713728.

18. Sluiter A., Hames B., Ruiz R., Scarlata C., Sluiter J., Templeton D., Crocker D. (2011). "Determination of structural carbohydrates and lignin in biomass," Technical Report, NREL/TP-510-42618, Colorado, United States.

19. Wang, Q., Wang, W., Tan, X., Zahoor, Chen, X., Guo, Y., Yu, Q. (2019). "Lowtemperature sodium hydroxide pretreatment for ethanol production from sugarcane bagasse without washing process," Bioresour. Technol., 291, 121844.
20. Wang, W., Wang X., Zhang, Y., Yu, Q., Tan, X., Zhuang X., Yuan, Z. (2020). "Effect of sodium hydroxide pretreatment on physicochemical changes and enzymatic hydrolysis of herbaceous and woody lignocelluloes," Ind. Crops Prod., 145, 112145.

21. Wunna, K., Nakasaki, K., Auresenia, J., Abella L., Gaspillo Pag-asa D. (2017). "Effect of alkali pretreatment on removal of lignin from sugarcane bagasse," Chem. Eng. Transc., 56, 1831-1836

22. Xu, F., Shi, Y. C., Wang, D. (2012). "Structural features and changes of lignocellulosic biomass during thermochemical pretreatments: a synchrotron X-ray scattering study on photoperiod-sensitive sorghum," Carbohydr. Polym, 88, 1149-1156. 